\title{
Mixed Convection in a Composite System Bounded by Vertical Walls
}

\author{
N. Srivastava ${ }^{1}$ and A. K. Singh ${ }^{2}$ \\ Department of Mathematics, Banaras Hindu University, Varanasi-221005, India \\ Email: ${ }^{1}$ neetusri_81@rediffmail.com, ${ }^{2}$ ashok@bhu.ac.in
}

(Received March 30, 2009; accepted September 6, 2009)

\begin{abstract}
A combined convection process between two parallel vertical infinite walls, containing an incompressible viscous fluid layer and a fluid saturated porous layer has been presented analytically. There is a vertical axial variation of temperature in the upward direction along the walls. The Brinkman extended Darcy model is applied to describe the momentum transfer in the porous region. The viscosity of the fluid layer and the effective viscosity of the porous layer are assumed to be different. Also the thermal conductivities of both fluid and porous layers are assumed to be different. The graphs and tables have been used to distinguish the influence of distinct parameters on the velocity and skin-friction. It is determined that the velocity is intensified on making greater the temperature difference between the walls while increment in the viscosity ratio (porous/fluid) parameter diminishes the velocity of the fluid. It has been observed that the numerical values of the skin-frictions have an increasing tendency with the increment in the values of temperature difference between the walls while decreasing tendency with the increment in the viscosity ratio parameter (porous/fluid).
\end{abstract}

Keywords: Mixed convection, composite system, effective viscosity, porous media, Brinkman model

\section{NOMENCLATURE}

$\begin{array}{ll}A & \begin{array}{l}\text { temperature gradient } \\ \text { along the wall }\end{array} \\ D a & \begin{array}{l}\text { Darcy number } \\ d^{\prime}\end{array} \\ d & \begin{array}{l}\text { distance of interface } \\ \text { distance of interface in } \\ \text { non-dimensional form }\end{array} \\ g & \begin{array}{l}\text { acceleration due to } \\ \text { gravity }\end{array} \\ H & \begin{array}{l}\text { distance between the } \\ \text { vertical walls }\end{array} \\ k^{\prime} & \begin{array}{l}\text { permeability of the porous } \\ \text { medium }\end{array} \\ P^{\prime} & \begin{array}{l}\text { pressure } \\ \text { constant including } \\ \text { pressure gradient term }\end{array} \\ R a & \begin{array}{l}\text { Rayleigh number } \\ \text { ratio of thermal } \\ R c\end{array} \\ R v & \begin{array}{l}\text { conductivities } \\ \text { ratio of effective viscosity } \\ \text { to the dynamic viscosity }\end{array} \\ T_{0}^{\prime} & \text { reference temperature } \\ T_{1}^{\prime} & \text { temperature at the wall } y^{\prime}=0\end{array}$
$u^{\prime} \quad$ velocity along the $x^{\prime}$-direction
$u \quad$ velocity along $x$-direction in non-
dimensional form
$x^{\prime} \quad$ vertical coordinate
$x \quad$ vertical coordinate in non- dimensional
form
$y^{\prime} \quad$ horizontal coordinate
$y$ horizontal coordinate in non- dimensional form

\section{Greek symbols}

$\alpha \quad$ thermal diffusivity

$\beta \quad$ coefficient of thermal expansion

$\mu_{f} \quad$ dynamic viscosity of the fluid

$\mu_{e f f} \quad$ effective viscosity of the porous region

$\theta$ temperature in non-dimensional form

$\rho \quad$ density
Subscripts
$f \quad$ fluid layer
$p \quad$ porous layer 


\section{INTRODUCTION}

The phenomena of mixed or combined convection arise when both free and forced convection simultaneously occur. Free convection means the motion which arises due to buoyancy effects while forced convection results due to any external force. An analytical solution of mixed convective flow between vertical parallel walls for higher Rayleigh number has been presented by Beckett and Friend (1984). A study of mixed convection flows between parallel plate channels has been presented by Aung and Worku (1986). An exact analytical solution of mixed convective flow on a permeable vertical cylinder in a saturated porous medium has been given by Ramanaiah and Malarvizhi (1990). Singh et al. (1993) have presented numerically the three dimensional free convection in a cavity as a result of side heating. An analytical study of natural convective flow in a composite system containing fluid and porous layers between two vertical walls has been presented by Paul et al. (1998). Paul et al. (1999) have further extended in case of unsteady natural convective flow.

The problem of mixed convection in a porous medium bounded by two vertical walls has been done by Mishra et al. (2002). Nobari and Beshkani (2007) have studied numerically the mixed convective flow in a vertical channel by applying finite difference method based on projection algorithm. The mixed convective flow between vertical parallel plates has been solved numerically by Guillet et al. (2007). A numerical study of mixed convective flow in rotating ducts has been done by Chiu et al. (2007). Ahmad et al. (2008) has investigated numerically the mixed convection along vertical thin needles. An analytical solution as well as a numerical solution is obtained by Barletta (2008) for the mixed convective flow in an inclined tube. The problem of unsteady turbulent flow and mixed convection has been presented numerically by Perng and $\mathrm{Wu}$ (2008). A numerical solution of mixed convective flow across a confined square cylinder has been acquired by Dhiman et al. (2008). A numerical result of the problem of mixed convective flow along a vertical slender cylinder has been given by Singh and Roy (2008) and the corresponding result for unsteady mixed convective flow has been shown by Singh et al. (2008). The problem of mixed convection of a nano fluid containing water and $\mathrm{Al}_{2} \mathrm{O}_{3}$ in horizontal and inclined tubes with constant heat flux has been examined numerically by Akbari et al. (2008).

Zanchini (2008) has given an analytical solution of mixed convective flows in a vertical annulus having constant wall temperatures. For the mixed convective flow of a viscoelastic fluid over a horizontal circular cylinder a numerical result has been presented by Anwar et al. (2008). Using Keller-box method, a numerical study of mixed convective flow in a porous medium has been done by Ishak et al. (2008). The problem of mixed convective flow from a wavy surface has been investigated experimentally by Kuhn and Rohr (2008). The study of turbulent mixed convection from vertical, parallel plate channels is discussed by Balaji et al. (2008) by applying asymptotic considerations. The effect of surface mass transfer on mixed convective flows is exhibited by giving numerical solution by Datta et al. (2008). Mohammed (2008) has given an experimental result of mixed convective flow in a vertical circular tube under constant heat flux boundary conditions. The problem of mixed convection in a rectangular enclosure is numerically solved by Saha et al. (2008) using finite element method. Sharma and Singh (2009) have shown the effects of variable thermal conductivity and heat source/sink on flow of a viscous incompressible electrically conducting fluid past a semi-infinite flat plate by using shooting method. A numerical solution is carried out by Mahanti and Gaur (2009) in order to show the effect of varying viscosity and thermal conductivity on steady natural convective flow of a viscous incompressible fluid by applying the Runge-Kutta fourth order method with shooting technique.

The study of mixed convection in a composite system has its importance in various fields such as aeronautical engineering, petroleum reservoir engineering, the technologies of paper, geothermal energy, storage system etc. The motive of contemplating this study is to present an analytical solution of mixed convection between two vertical walls containing a fluid and a porous material when the temperature of the walls varies vertically upward. Brinkman extended Darcy model is used to model the flow in porous region. For fully developed laminar flow, the velocity has only one component in vertical direction. The viscosity of the fluid layer and the effective viscosity of the porous layer are taken to be different. Three different analytical solutions of the model have been obtained depending on the values of Darcy number, viscosity ratio parameter, thermal conductivity ratio parameter and Rayleigh number. Finally effects of various physical parameters have been shown by using graphs and tables.

\section{Mathematical Formulation}

In the given problem a steady fully developed laminar free convective flow between two infinite vertical walls filled with a fluid layer and a fluid saturated porous layer is considered as shown in Fig. 1. The interface of fluid and porous layers is taken permeable so that fluid can flow from one layer to other. The $x^{\prime}$-axis is taken in the vertical direction while $y^{\prime}$-axis is taken in the horizontal direction. The walls at $y^{\prime}=0$ and $y^{\prime}=H$ are maintained at the temperatures $T_{0}+A x^{\prime}$ and $T_{1}+A x^{\prime}$ respectively, where $x^{\prime}$ is the distance measured vertically in the upward direction. Under usual Boussinesq's approximation, the governing equations in the reference of the considered problem in non-dimensional form are derived as follows:

For fluid region (Beckett and Friend (1984)):

$$
\begin{aligned}
& \frac{d^{2} u_{f}}{d y^{2}}+R a \theta_{f}=-1, \\
& \frac{d^{2} \theta_{f}}{d y^{2}}-u_{f}=0,
\end{aligned}
$$

For porous region (Mishra et al. (2002)):

$R v \frac{d^{2} u_{p}}{d y^{2}}+R a \theta_{p}-\frac{1}{D a} u_{p}=-1$, 
$R c \frac{d^{2} \theta_{p}}{d y^{2}}-u_{p}=0$.

The corresponding boundary and matching conditions in non-dimensional form are acquired as follows (Singh et al. (1993)):

$$
\begin{aligned}
& \text { at } y=0, \quad u_{f}=0, \quad \theta_{f}=0 \text {, } \\
& \text { at } y=1, \quad u_{p}=0, \quad \theta_{p}=\frac{T_{1}{ }^{\prime}-T_{0}{ }^{\prime}}{A H Q}=Q \text {, } \\
& \text { at } y=d, \quad u_{f}=u_{p}, \quad \frac{d u_{f}}{d y}=R v \frac{d u_{p}}{d y}, \\
& \text { at } y=d, \quad \theta_{f}=\theta_{p}, \quad \frac{d \theta_{f}}{d y} R c \frac{d \theta_{p}}{d y} \text {. }
\end{aligned}
$$

The non-dimensional quantities used in the above equations are obtained as follows:

$$
\begin{array}{lrl}
y=\frac{y^{\prime}}{d}, & u_{f}=\frac{u_{f}^{\prime} H}{Q \alpha_{f}}, & u_{p}=\frac{u_{p}^{\prime} H}{Q \alpha_{f}}, \\
\theta_{f}=\frac{\theta_{f}^{\prime}}{A H Q}, & \theta_{p}=\frac{\theta_{p}^{\prime}}{A H Q}, & R v=\frac{\mu_{e f f}}{\mu_{f}}, \\
D a=\frac{K^{\prime}}{H^{2}}, & R c=\frac{\alpha_{p}}{\alpha_{f}}, & R a=\frac{\rho g A \beta H^{4}}{\alpha_{f} \mu_{f}}, \\
Q=\frac{\rho H^{3}}{\alpha_{f} \mu_{f}}\left\{\left(-\frac{1}{\rho} \frac{d P^{\prime}}{d x^{\prime}}+g\right)+g \beta A x^{\prime}\right\} .
\end{array}
$$

Eliminating $\theta_{f}$ from Eqs. (1) and (2), we get a fourth order differential equation in $u_{f}$ as

$\frac{d^{4} u_{f}}{d y^{4}}+R a u_{f}=0$

while eliminating $\theta_{p}$ from Eqs. (3) and (4) a fourth order differential equation in $u_{p}$ is obtained as

$\frac{d^{4} u_{p}}{d y^{4}}-\frac{1}{D a} \frac{d^{2} u_{p}}{d y^{2}}+\frac{R a}{R c} u_{p}=0$,

whose auxiliary roots are obtained as given below:

$m_{1}, m_{2}= \pm \sqrt{\frac{1+S}{2 D a R v}}$,

$m_{3}, m_{4}= \pm \sqrt{\frac{1-S}{2 D a R v}}$

where

$S=\sqrt{1-\frac{4 R a R v D a^{2}}{R c}}$.

It is obvious from the auxiliary roots described in Eq. (9), that the solution for the velocity and temperature fields depends on the values of $R a, R v$, $D a$ and $R c$ and there arises three different cases which are as follows:

Case1. When $1-\frac{4 R a R v D a^{2}}{R c}>0$;

In this case $S$ will be a real number. Solving Eqs. (7) and (8) with their proper boundary conditions the solution for $u_{f}, \theta_{f}, u_{p}$ and, $\theta_{p}$ are obtained as given below:

$$
\begin{aligned}
u_{f}= & e^{N y}\left(C_{1} \cos N y+C_{2} \sin N y\right) \\
& +e^{-N y}\left(C_{3} \cos N y+C_{4} \sin N y\right) \\
\theta_{f} & =-\frac{1}{R a}-\frac{2 N^{2}}{R a}\left[e^{N y}\left(C_{2} \cos N y-C_{1} \sin N y\right)\right. \\
& \left.+e^{-N y}\left(C_{3} \sin N y-C_{4} \cos N y\right)\right], \\
u_{p}= & \left(C_{5} \cosh h_{1} y+C_{6} \sinh h_{1} y\right) \\
& +\left(C_{7} \cosh h_{2} y+C_{8} \sinh h_{2} y\right) \\
\theta_{p} & =-\frac{1}{R a}-\frac{R v}{R a}\left[C_{5} h_{1}^{2} \cosh \mathrm{h}_{1} \mathrm{y}+C_{6} h_{1}^{2} \sinh \mathrm{h}_{1} \mathrm{y}\right. \\
& \left.+C_{7} h_{2}^{2} \cosh \mathrm{h}_{2} \mathrm{y}+C_{8} h_{2}^{2} \sinh h_{2} y\right]+\frac{1}{D a R a} \\
& {\left[C_{5} \cosh \left(h_{1} y\right)+C_{6} \sinh \left(h_{1} y\right)+C_{7} \cosh \left(h_{2} y\right)\right.} \\
& \left.+C_{8} \sinh \left(h_{2} y\right)\right] .
\end{aligned}
$$

The above solutions are valid only for $0<S<1$.

Case2. When $1-\frac{4 R a R v D a^{2}}{R c}=0$;

In this case, the velocity and temperature fields in the fluid and porous region with the suitable boundary conditions are derived as

$$
\begin{aligned}
u_{f}= & e^{N y}\left(C_{1} \cos N y+C_{2} \sin N y\right) \\
& +e^{-N y}\left(C_{3} \cos N y+C_{4} \sin N y\right), \\
\theta_{f}= & -\frac{1}{R a}-\frac{2 N^{2}}{R a}\left[e^{N y}\left(C_{2} \cos N y-C_{1} \sin N y\right)\right. \\
& \left.+e^{-N y}\left(C_{3} \sin N y-C_{4} \cos N y\right)\right], \\
u_{p}= & \left(C_{5}+C_{6} y\right) \cosh m y \\
& +\left(C_{7}+C_{8} y\right) \sinh m y, \\
\theta_{p}= & -\frac{1}{R a}-\frac{R v}{R a}\left[\left\{m^{2}\left(C_{1}+C_{2} y\right)+2 m C_{4}\right\}\right. \\
& \left.\cosh m y+\left\{m^{2}\left(C_{3}+C_{4} y\right)+2 m C_{2}\right\} \sinh m y\right] \\
& +\frac{1}{\text { DaRa }}\left[\left(C_{1}+C_{2} y\right) \cosh m y+\left(C_{3}+C_{4} y\right)\right. \\
& \sinh m y] .
\end{aligned}
$$

Case3. When $1-\frac{4 R a R v D a^{2}}{R c}<0$;

$\mathrm{S}$ will be imaginary in this case. The expressions for the velocity and temperature fields in the fluid and porous region with the corresponding boundary conditions are

$$
\begin{aligned}
u_{f} & =e^{N y}\left(C_{1} \cos N y+C_{2} \sin N y\right) \\
& +e^{-N y}\left(C_{3} \cos N y+C_{4} \sin N y\right), \\
\theta_{f} & =-\frac{1}{R a}-\frac{2 N^{2}}{R a}\left[e^{N y}\left(C_{2} \cos N y-C_{1} \sin N y\right)\right. \\
& \left.+e^{-N y}\left(C_{3} \sin N y-C_{4} \cos N y\right)\right],
\end{aligned}
$$




$$
\begin{aligned}
u_{p}= & e^{\alpha y}\left(C_{5} \cos \beta y+C_{6} \sin \beta y\right) \\
& +e^{-\alpha y}\left(C_{7} \cos \beta y+C_{8} \sin \beta y\right), \\
\theta_{p}= & -\frac{1}{R a}+\frac{1}{D a R a}\left[e^{a y} \beta\left\{C_{5} \cos \beta y+C_{6} \sin \beta y\right\}\right. \\
& \left.+e^{-a y}\left\{C_{7} \cos \beta y+C_{8} \sin \beta y\right\}\right]-\frac{R v}{R a}\left[C_{5} e^{a y}\right. \\
& \left\{\left(\alpha^{2}-\beta^{2}\right) \cos \beta y-2 \alpha \beta \sin \beta y\right\}+C_{6} e^{a y} \\
& \left\{\left(\alpha^{2}-\beta^{2}\right) \sin \beta y+2 \alpha \beta \cos \beta y\right\}+C_{7} e^{-a y} \\
& \left\{\left(\alpha^{2}-\beta^{2}\right) \cos \beta y+2 \alpha \beta \sin \beta y\right\}+C_{8} e^{-a y} \\
& \left.\left\{\left(\alpha^{2}-\beta^{2}\right) \sin \beta y-2 \alpha \beta \cos \beta y\right\}\right] .
\end{aligned}
$$

By using the Eqs. (10), (12), (14), (16), (18) and (20), expressions for the skin frictions in all three cases are derived as follows:

$$
\begin{aligned}
& \text { Case 1: When } 1-\frac{4 R a R v D a^{2}}{R c}>0 \text {; } \\
& \tau_{1}=\left(\frac{d u_{f}}{d y}\right)_{y=0}=N\left(C_{1}+C_{2}+C_{4}-C_{3}\right), \\
& \tau_{2}=-\left(\frac{d u_{p}}{d y}\right)_{y=1} \\
& =-\left(C_{5} h_{1} \sinh h_{1}+C_{6} h_{1} \cosh h_{1}\right) \\
& +\left(C_{7} h_{2} \sinh h_{2}+C_{8} h_{2} \cosh h_{2}\right) \text {. }
\end{aligned}
$$

Case 2: When $1-\frac{4 R a R v D a^{2}}{R c}=0$;

$$
\begin{aligned}
\tau_{1}= & -\left(\frac{d u_{f}}{d y}\right)_{y=0} \\
= & -N\left(C_{1}+C_{2}+C_{4}-C_{3}\right), \\
\tau_{2}= & \left(\frac{d u_{p}}{d y}\right)_{y=1} \\
= & \left\{\left(C_{5}+C_{6}\right) m+C_{8}\right\} \sinh m \\
& +\left\{\left(C_{7}+C_{8}\right) m+C_{6}\right\} \cosh m .
\end{aligned}
$$

Case 3: When $1-\frac{4 R a R v D a^{2}}{R c}<0$;

$\tau_{1}=\left(\frac{d u_{f}}{d y}\right)_{y=0}=N\left(C_{1}+C_{2}+C_{4}-C_{3}\right)$,

$\tau_{2}=-\left(\frac{d u_{p}}{d y}\right)_{y=1}$

$=e^{\alpha}\left\{C_{5}(\alpha \cos \beta-\beta \sin \beta)+C_{6}(\alpha \sin \beta+\right.$ $\beta \cos \beta-e \alpha C 8 \beta \cos \beta-\alpha \sin \beta-C 7 \beta \sin \beta+\alpha \cos \beta$ (27)

The parameters used in the above equations are mentioned in the appendix.

\section{Results AND Discussion}

The influence of different physical parameters on the velocity field is depicted in the Figs. 2-3 for the case $S>0$ while in the Figs. 4-6 for $S=0$ and finally for the case $S<0$ in the Figs. 7-11. In Fig. 2 the effect of thermal conductivity ratio parameter $R c$ and viscosity ratio parameter $R v$ on the velocity field is shown. From the figure it can be clearly observed that the velocity is showing decreasing behavior with the increment in the values of $R v$ for all the values of Darcy number and this is expected due to impact of more viscous force. For $D a=10^{-2}$, we can see that the effect of viscosity ratio parameter is negligible in the porous region. There is a decrement in the velocity on increasing the values of $R c$ in the fluid region while there is an increment in the velocity on increasing the values of $R c$ in the porous region. This phenomenon may occur due to more diffusion of heat in porous layer than fluid layer. In Fig. 3, the impact of pressure gradient constant $Q$, on the velocity field is hold to view. By examining the figure it has been noticed that the velocity becomes greater when the values of $Q$ grow i.e., when the temperature difference between two walls is increasing. This is attributed to fact that velocity increases due to increment in the buoyancy force. In Fig. 4, the effect on the velocity field for the different values of $R c, R v$ and $R a$ has been displayed when $S=0$. The figure manifestly notifies that on increasing the value of $R v$, the velocity decreases for all the values of Darcy number.

In Fig. 5, the relation between the fluid layer width $d$ and the velocity has been presented to view. The figure completely notifies that with the increment in the fluid layer width $d$ the velocity has growing nature for all the values of Darcy number. But for $D a=.05$, in the porous region there is a negligible influence of increment in fluid layer width. In Fig. 6, the variation in the velocity due to variation in the values of $Q$ has been shown. It is clear from the figure that when $Q=0$, that is when the temperature difference between two walls is zero then the flow is in the upward direction while for $Q>0$, the flow is in the reverse direction.

In Fig. 7, the velocity profiles are shown for different values of $R c$ and $R v$. It is remarkable from the figure that the effect of $R v$ on the velocity is to decrease it for all considered values of Darcy number. In this case also the velocity has a decreasing tendency in the fluid region while it is showing an increasing nature in the porous region on increasing the values of $R c$. Also velocity is more apparent for $D a=10^{-1}$ than that of $D a=10^{-2}$. In Fig. 8, the effect of Rayleigh number $R a$ on the velocity field has been exhibited. It is quite remarkable from the figure that there is a decrement in the velocity due to increment in the values of $R a$. In Fig. 8, the relation of velocity with the fluid layer width has been hold to view. From the figure it can be easily seen that the velocity varies in an increasing manner on increasing the fluid layer width. Figures 10 and 11 are given to describe the effect of $Q$ on the velocity when $Q$ hold the values close to zero and when $Q$ hold the values greater than zero respectively. From both the Figs. 9 and 10, it is obvious that the velocity has increasing tendency with the increment in the values of $Q$ for the all values of Darcy number.

At last, the numerical values of the skin-friction are computed on both the walls. Here, $\tau_{1}$ and $\tau_{2}$ represent 
the numerical values of skin-friction on the walls $y=0$ and $y=1$ respectively and they are given in Tables 1,2 and 3 for the different three cases $S>0, S=0$, and $S<0$ respectively. From the Table 1, it is observed that on increasing the values of $R v$ and $R a$ both $\tau_{1}$ and $\tau_{2}$ are decreasing while increasing due to increment in the values of Darcy number, fluid layer width $d$ and pressure gradient constant $Q$. The increment in the values of $R c$ makes decrement in $\tau_{1}$ while it makes increment in $\tau_{2}$ for all the values of Darcy number. From Table 2, it can be clearly viewed that the values of both $\tau_{1}$ and $\tau_{2}$ increase with the fluid layer width $d$ and pressure gradient constant $Q$. From Table 3, it can be notified that both $\tau_{1}$ and $\tau_{2}$ have increasing tendency when the Darcy number $D a$, fluid layer width $d$ and pressure gradient constant $Q$, tend to increment. With the increment in the values of $R c, \tau_{1}$ decreases while $\tau_{2}$ increases. In this case, both $\tau_{1}$ and $\tau_{2}$ are showing decreasing behavior on increasing the values of $R v$ and $R a$.

\section{Conclusion}

An analytical solution of mixed convection in a composite system containing a fluid layer and a porous layer bounded by vertical walls has been attained. It is resolved from the discussion that the velocity in both fluid and porous regions has decreasing tendency with the viscosity ratio parameter for all the cases which is due to fact that velocity decreases due to more viscous forces. The effect of the thermal conductivity ratio parameter is to enhance the velocity in the porous region while to diminish the velocity in the fluid region for all the values of Darcy number. Lastly, it has been concluded that numerical values of the skin friction exhibit an increasing behavior with the increment in the values of temperature difference between the walls, Darcy number and fluid layer width while the numerical values of skin friction are decreasing due to the viscosity ratio parameter and Rayleigh number.

\section{REFERENCES}

Ahmad, S., N.M. Arifin, R. Nazar and I. Pop (2008). Mixed convection boundary layer flow along vertical thin needless: Assisting and opposing flows. International Communications in Heat and Mass Transfer 35, 157-162.

Akbari, M., A. Behzadmehr and F. Shahraki (2008). Fully developed mixed convection in horizontal and inclined tubes with uniform heat flux using nanofluid. International Journal of Heat and Fluid Flow 29, 545-556.

Anwar, I., A. Norsarahaida and I. Pop (2008). Mixed convection boundary layer flow of a viscoelastic fluid over a horizontal circular cylinder. International journal of Non-Linear Mechanics 43, 814-821.

Aung, W. and G. Worku (1986). Theory of fully developed, combined convection including flow reversal. Journal of Heat Transfer 108, 485-488.
Balaji, C., M. Holling and H. Herwig (2008). A temperature wall functions for turbulent mixed convection from vertical, parallel plate channels. International Journal of Thermal Sciences 47, 723-729.

Barletta, A. (2008). Parallel and non-parallel laminar mixed convection flow in an inclined tube: The effect of the boundary conditions. International Journal of Heat and Fluid Flow 29, 83-93.

Beckett, P.M. and I.E. Friend (1984). Combined natural and forced convection between parallel walls: Developing flow at higher Rayleigh numbers. International Journal of Heat and Mass Transfer 27, 611-621.

Chiu, H., J. Jang and W. Yan (2007). Combined mixed convection and radiation heat transfer in rectangular ducts rotating about a parallel axis. International Journal of Heat and Mass Transfer 50, 4229-4242.

Datta, P., S.V. Subhashini and R. Ravindran (2009). Influence of surface mass transfer on mixed convection flows over non-isothermal horizontal flat plates. Applied Mathematical Modelling 33, 1285-1294.

Dhiman, A.K., R.P. Chhabra and V. Eswaran (2008). Steady mixed convection across a confined square cylinder, International Communications in Heat and Mass Transfer 35, 47-55.

Guillet, C., T. Mare and C.T. Nguye (2007). Application of a non-linear local analysis method for the problem of mixed convection instability. International Journal of Non-Linear Mechanics 42, 981-988.

Ishak, A., R. Nazar and I. Pop (2008). Dual solutions in mixed convection flow near a stagnation point on a vertical surface in a porous medium. International Journal of Heat and Mass Transfer 51, 1150-1155.

Kuhn, S. and P.R.V. Rohr (2008). Experimental investigation of mixed convective flow over a wavy wall. International Journal of Heat and Fluid Flow 29, 94-106.

Mahanti, N.C. and P. Gaur (2009). Effects of varying viscosity and thermal conductivity on steady Free convective flow and heat transfer along an isothermal vertical plate in the presence of heat sink. Journal of Applied Fluid Mechanics 2, 23-28.

Mishra, A.K., T. Paul and A.K. Singh (2002). Mixed convection in a porous medium bounded by two vertical walls. Forschung im Ingenieurwesen 67, 198-205.

Mohammed, H.A. (2008). Laminar mixed convection heat transfer in a vertical circular tube under 
buoyancy-assisted and opposed flow. Energy Conservation and Management 49, 2006-2015.

Nobari, M.R.H. (2007). A numerical study of mixed convection in a vertical channel flow impinging on a horizontal surface. International Journal of Thermal Sciences 46, 989-997.

Paul, T., A.K. Singh and G.R. Thorpe (1999). Transient natural convection in a vertical channel partially filled with a porous medium. Journal of Mathematical Engineering in Industry 7, 441-455.

Paul, T., B.K. Jha and A.K. Singh (1998). Free convection between vertical walls partially filled with porous medium. Heat and Mass Transfer 33, 515-519.

Perng, S. and H. Wu (2008). Numerical investigation of mixed convective heat transfer for unsteady turbulent flow over heated blocks in a horizontal channel. International Journal of Thermal Sciences 47, 620-632.

Ramanaiah, G. and G. Malarvizhi (1990). Unified treatment of free and mixed convection on a permeable vertical cylinder in a saturated porous medium. Indian Journal of Technology 28, 604608 .

Saha, S., M.A.H. Mamun, M.Z. Hossain and A.K.M.S. Islam (2008). Mixed convection in an enclosure with different inlet and exit configurations. Journal of Applied Fluid Mechanics 1, 78-93.

Sharma, P.R. and G. Singh (2009). Effects of variable thermal conductivity and heat source/ sink on MHD flow near a stagnation point on a linearly stretching sheet. Journal of Applied Fluid Mechanics 2, 13-21.

Singh, A.K., E. Leonardi and G.R. Thorpe (1993). 3Dimensional natural convection in a confined fluid overlying a porous layer. ASME Journal of Heat Transfer 115, 631-638.

Singh, P.J. and S. Roy (2008). Mixed convection along a rotating vertical slender cylinder in an axial flow. International Journal of Heat and Mass Transfer 51, 717-723.

Singh, P.J., S. Roy and I. Pop (2008). Unsteady mixed convection from a rotating vertical slender cylinder in an axial flow. International Journal of Heat and Mass Transfer 51, 1423-1430.

Zanchini, E. (2008). Mixed convection with variable viscosity in a vertical annulus with uniform wall temperatures. International Journal of Heat and Mass Transfer 51, 30-40.

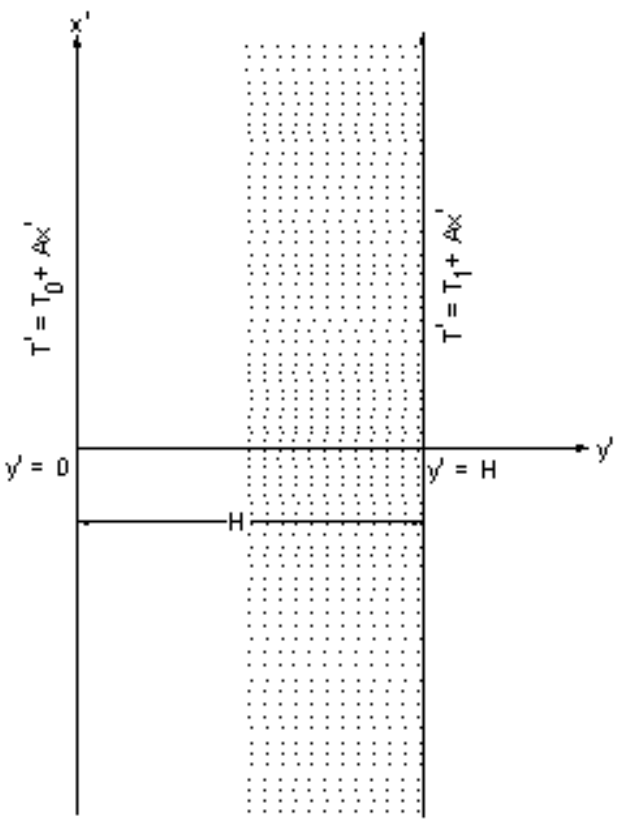

Fig. 1. Physical configuration of the model

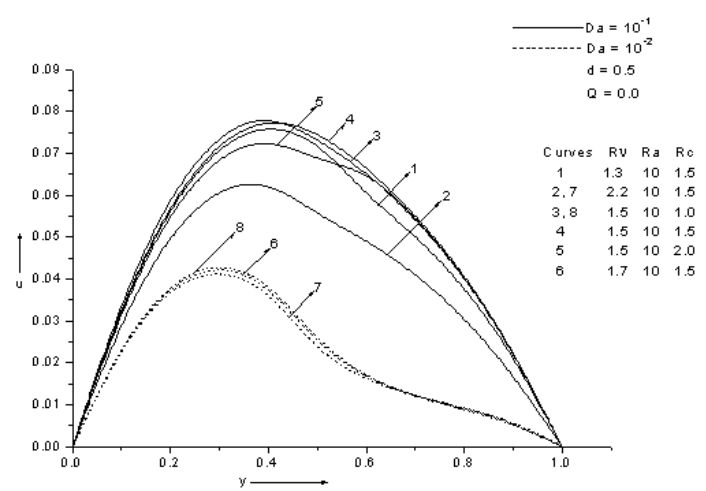

Fig. 2. Velocity profiles for different values of Rc and Rv (Case 1)

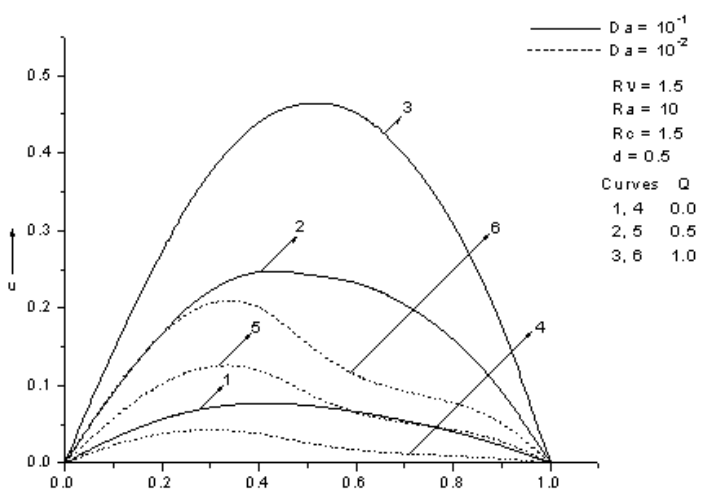

Fig. 3. Velocity profiles for different values $Q$ (Case 1) 


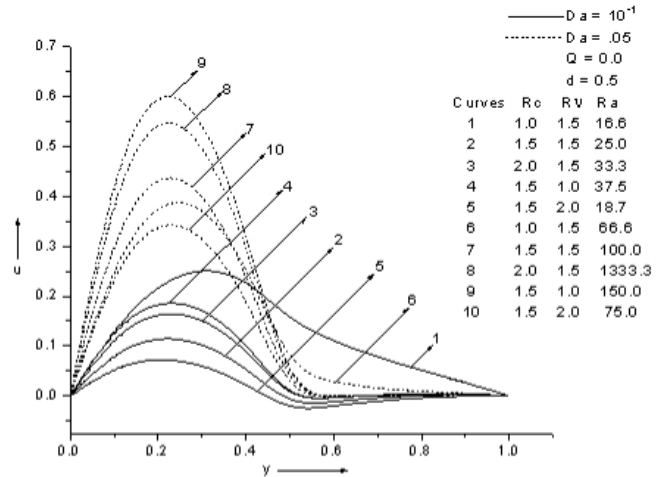

Fig. 4. Velocity profiles for different values of Rc, $\mathrm{Rv}$ and $\mathrm{Ra}$ (Case 2)

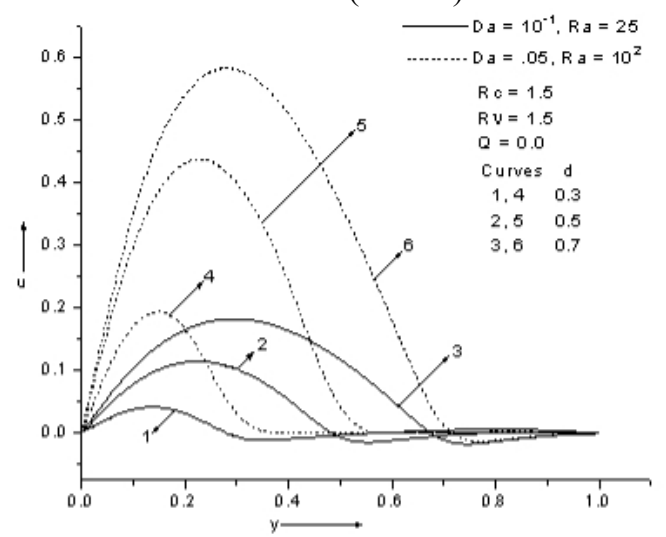

Fig. 5. Velocity profiles for different values of $d$ (Case 2)

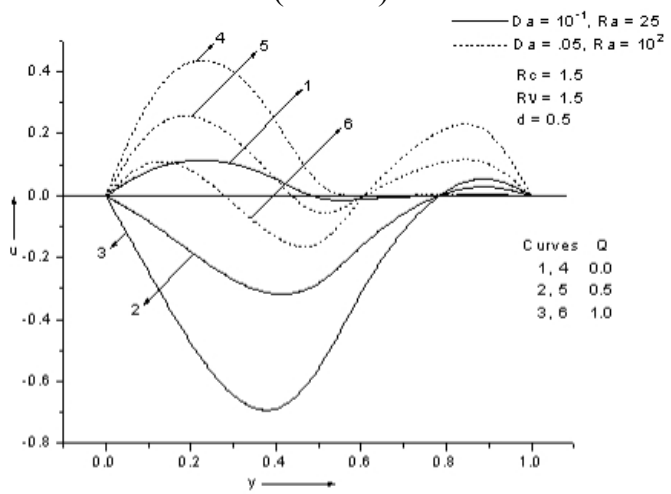

Fig. 6. Velocity profiles for different values of $Q$ (Case 2)

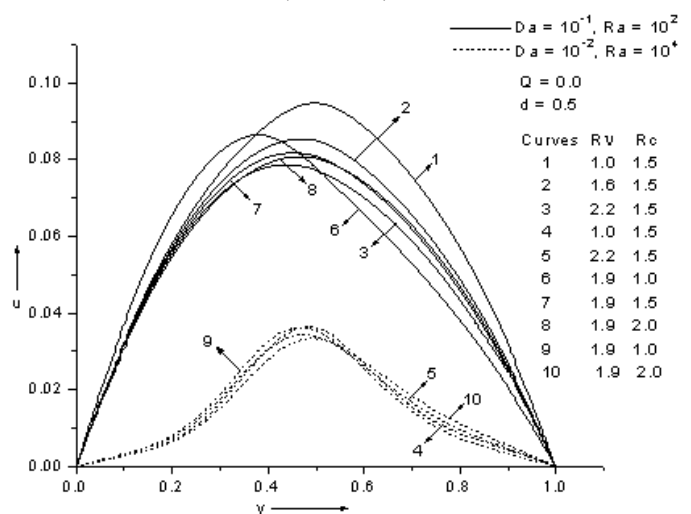

Fig. 7. Velocity profiles for different values of Rc and Rv (Case 3)

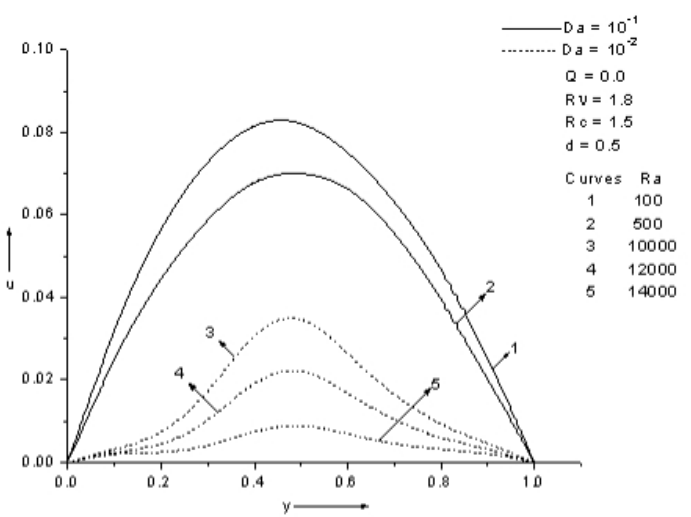

Fig. 8. Velocity profiles for different values of $\mathrm{Ra}$ (Case 3)

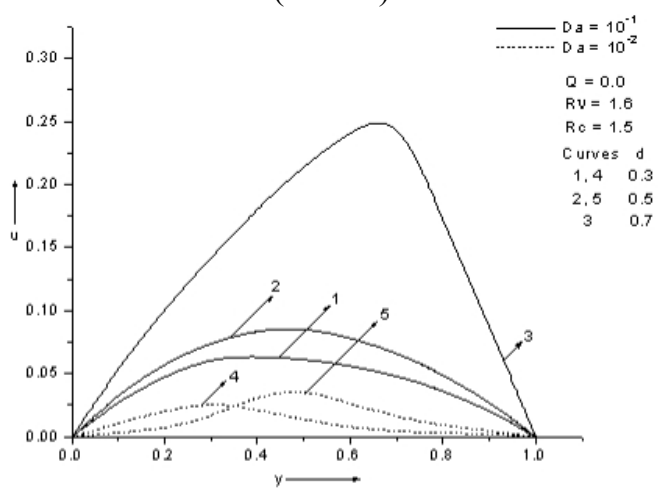

Fig. 9. Velocity profiles for different values of $d$ (Case 3)

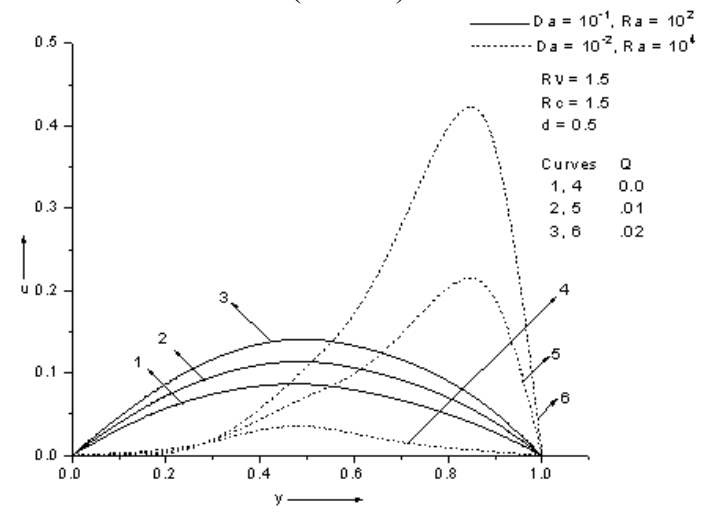

Fig. 10. Velocity profiles for different values of $\mathrm{Q}$ (Case 3)

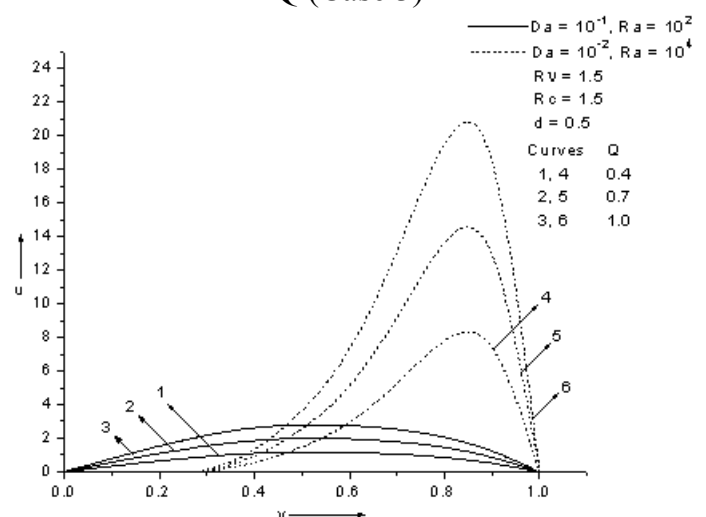

Fig. 11. Velocity profiles for different values of Q (Case 3) 
Numerical values of skin frictions

Table1 Case 1

\begin{tabular}{|c|c|c|c|c|c|c|c|}
\hline \multirow{1}{*}{$\boldsymbol{a}$} & $\mathrm{Q}$ & $\boldsymbol{R} \boldsymbol{a}$ & $\boldsymbol{R} \boldsymbol{v}$ & $\boldsymbol{R} \boldsymbol{c}$ & $\boldsymbol{d}$ & $\tau_{1}$ & $\tau_{2}$ \\
\hline $10^{-1}$ & 0.0 & 10 & 1.3 & 1.5 & 0.5 & 0.386669 & 0.254900 \\
& 0.0 & 10 & 1.8 & 1.5 & 0.5 & 0.386578 & 0.220868 \\
& 0.0 & 10 & 1.5 & 1.0 & 0.5 & 0.401966 & 0.260029 \\
& 0.0 & 10 & 1.5 & 2.0 & 0.5 & 0.380595 & 0.260339 \\
& 0.0 & 15 & 1.5 & 1.5 & 0.5 & 0.366278 & 0.236119 \\
& 0.0 & 22 & 1.5 & 1.5 & 0.5 & 0.362220 & 0.225121 \\
& 0.0 & 10 & 1.5 & 1.5 & 0.3 & 0.348182 & 0.215806 \\
& 0.0 & 10 & 1.5 & 1.5 & 0.7 & 0.422484 & 0.280347 \\
& 0.5 & 10 & 1.5 & 1.5 & 0.5 & 0.998385 & 1.153310 \\
& 1.0 & 10 & 1.5 & 1.5 & 0.5 & 1.540800 & 2.201330 \\
& 0.0 & 10 & 1.3 & 1.5 & 0.5 & 0.298580 & 0.091616 \\
& 0.0 & 10 & 1.8 & 1.5 & 0.5 & 0.293953 & 0.079290 \\
& 0.0 & 10 & 1.5 & 1.0 & 0.5 & 0.296468 & 0.085167 \\
& 0.0 & 10 & 1.5 & 2.0 & 0.5 & 0.296415 & 0.086007 \\
& 0.0 & 15 & 1.5 & 1.5 & 0.5 & 0.294816 & 0.085056 \\
& 0.0 & 22 & 1.5 & 1.5 & 0.5 & 0.290567 & 0.084347 \\
& 0.0 & 10 & 1.5 & 1.5 & 0.3 & 0.206724 & 0.081821 \\
& 0.0 & 10 & 1.5 & 1.5 & 0.7 & 0.393193 & 0.116738 \\
& 0.5 & 10 & 1.5 & 1.5 & 0.5 & 0.665799 & 0.464376 \\
& 1.0 & 10 & 1.5 & 1.5 & 0.5 & 1.034200 & 0.838864 \\
\hline \multirow{11}{*}{-2}
\end{tabular}

Table2 Case 2

\begin{tabular}{|c|c|c|c|c|c|c|c|}
\hline \multirow{2}{*}{$\boldsymbol{a}$} & $\mathrm{Q}$ & $\boldsymbol{R} \boldsymbol{a}$ & $\boldsymbol{R} \boldsymbol{v}$ & $\boldsymbol{R} \boldsymbol{c}$ & $\boldsymbol{d}$ & $\tau_{1}$ & $\tau_{2}$ \\
\hline $10^{-1}$ & 0.0 & 25 & 1.5 & 1.5 & 0.3 & 0.206240 & 0.870637 \\
& 0.0 & 25 & 1.5 & 1.5 & 0.5 & 0.392751 & 1.056768 \\
& 0.0 & 25 & 1.5 & 1.5 & 0.7 & 0.964422 & 1.627459 \\
& 0.5 & 25 & 1.5 & 1.5 & 0.5 & 0.512209 & 1.323730 \\
& 1.0 & 25 & 1.5 & 1.5 & 0.5 & 0.631668 & 1.590671 \\
& 0.0 & 16.6 & 1.5 & 1.0 & 0.5 & 0.166228 & 0.950956 \\
& 0.0 & 33.3 & 1.5 & 2.0 & 0.5 & 2.100763 & 2.803604 \\
& 0.0 & 18.7 & 2.0 & 1.5 & 0.5 & 2.292225 & 1.237157 \\
& 0.0 & 20.8 & 1.5 & 1.8 & 0.5 & 0.886599 & 1.533033 \\
& 0.0 & 100 & 1.5 & 1.5 & 0.3 & 0.560225 & 0.532392 \\
& 0.0 & 100 & 1.5 & 1.5 & 0.5 & 0.727771 & 0.551913 \\
& 0.0 & 100 & 1.5 & 1.5 & 0.7 & 0.974949 & 0.578088 \\
& 0.5 & 100 & 1.5 & 1.5 & 0.5 & 0.929358 & 0.687462 \\
& 1.0 & 100 & 1.5 & 1.5 & 0.5 & 1.130945 & 0.823012 \\
& 0.0 & 133.3 & 1.5 & 2.0 & 0.5 & 0.537431 & 0.488496 \\
& 0.0 & 20.8 & 1.5 & 1.8 & 0.5 & 0.563640 & 0.497770 \\
\hline \multirow{6}{*}{05}
\end{tabular}

Table 3 Case 3

\begin{tabular}{|c|c|c|c|c|c|c|c|}
\hline $\boldsymbol{D a}$ & $\mathrm{Q}$ & $\boldsymbol{R a}$ & $\boldsymbol{R} \boldsymbol{R} \boldsymbol{c}$ & $\boldsymbol{d}$ & $\tau_{1}$ & $\tau_{2}$ \\
\hline $10^{-1}$ & 0.0 & 100 & 1.0 & 1.5 & 0.5 & 0.383112 & 0.386109 \\
& 0.0 & 100 & 2.0 & 1.5 & 0.5 & 0.379016 & 0.283202 \\
& 0.0 & 100 & 1.5 & 1.0 & 0.5 & 0.471167 & 0.236866 \\
& 0.0 & 100 & 1.5 & 2.0 & 0.5 & 0.372853 & 0.335647 \\
& 0.0 & 500 & 1.5 & 1.5 & 0.5 & 0.309017 & 0.226889 \\
& 0.0 & 900 & 1.5 & 1.5 & 0.5 & 0.291173 & 0.198156 \\
& 0.0 & 100 & 1.5 & 1.5 & 0.3 & 0.326804 & 0.249637 \\
& 0.0 & 100 & 1.5 & 1.5 & 0.7 & 0.587187 & 0.941567 \\
& 0.5 & 100 & 1.5 & 1.5 & 0.5 & 4.314726 & 8.213052 \\
& 1.0 & 100 & 1.5 & 1.5 & 0.5 & 8.246530 & 16.104570 \\
& 0.0 & 10000 & 1.0 & 1.5 & 0.5 & 0.061075 & 0.078267 \\
& 0.0 & 10000 & 2.0 & 1.5 & 0.5 & 0.063788 & 0.061201 \\
& 0.0 & 10000 & 1.5 & 1.0 & 0.5 & 0.062881 & 0.054239 \\
& 0.0 & 10000 & 1.5 & 2.0 & 0.5 & 0.061432 & 0.076134 \\
& 0.0 & 12000 & 1.5 & 1.5 & 0.5 & 0.059315 & 0.056257 \\
& 0.0 & 14000 & 1.5 & 1.5 & 0.5 & 0.060947 & 0.047887 \\
& 0.0 & 1000 & 1.5 & 1.5 & 0.3 & 0.139523 & 0.047492 \\
\hline
\end{tabular}


N. Srivastava and A. K. Singh / JAFM, Vol. 3, No. 2, pp. 65-75, 2010.

\section{Appendix}

Case 1:

$$
\begin{array}{lll}
S=\sqrt{1-\frac{4 R a R v D a^{2}}{R c},} & A=\frac{1+S}{2 D a R v}, \\
N=\sqrt{\frac{\sqrt{R a}}{2}}, & B=\frac{1-S}{2 D a R v}, \\
h_{1}=\sqrt{A}, & h_{2}=\sqrt{B}, \\
h_{3}=h_{1} d, & h_{4}=h_{2} d, \\
h_{6}=N d, & h_{7}=2 N^{2}, \\
r_{1}=\cosh \left(h_{1}\right), & r_{2}=\sinh \left(h_{1}\right), \\
r_{3}=\cosh \left(h_{2}\right) & r_{4}=\sinh \left(h_{2}\right), \\
r_{5}=e^{h_{6}} \cos \left(h_{6}\right), & r_{6}=e^{h_{6}} \sin \left(h_{6}\right), \\
r_{7}=e^{-h_{6}} \cos \left(h_{6}\right), & r_{8}=e^{-h_{6}} \sin \left(h_{6}\right),
\end{array}
$$$$
r_{12}=\sinh \left(h_{4}\right) \text {, }
$$$$
r_{13}=N e^{h_{6}}\left\{\cos \left(h_{6}\right)-\sin \left(h_{6}\right)\right\} \text {, }
$$$$
r_{14}=N e^{h_{6}}\left\{\cos \left(h_{6}\right)+\sin \left(h_{6}\right)\right\} \text {, }
$$$$
r_{15}=-N e^{-h_{6}}\left\{\cos \left(h_{6}\right)+\sin \left(h_{6}\right)\right\} \text {, }
$$$$
r_{16}=N e^{-h_{6}}\left\{\cos \left(h_{6}\right)-\sin \left(h_{6}\right)\right\} \text {, }
$$$$
r_{17}=R v h_{1} \sinh \left(h_{3}\right) \text {, }
$$$$
r_{18}=R v h_{1} \cosh \left(h_{3}\right) \text {, }
$$$$
r_{19}=R v h_{2} \sinh \left(h_{4}\right) \text {, }
$$$$
r_{20}=R v h_{2} \cosh \left(h_{4}\right) \text {, }
$$$$
r_{21}=-\frac{1}{h_{7}},
$$$$
r_{22}=h_{1}^{2} \cosh \left(h_{1}\right) \text {, }
$$$$
r_{23}=h_{1}^{2} \sinh \left(h_{1}\right) \text {, }
$$$$
r_{24}=h_{2}^{2} \cosh \left(h_{2}\right) \text {, }
$$$$
r_{27}=\left(\frac{1}{D a}-R v h_{1}^{2}\right) \cosh \left(h_{3}\right) \text {, }
$$$$
r_{28}=\left(\frac{1}{D a}-R v h_{1}^{2}\right) \sinh \left(h_{3}\right),
$$$$
r_{29}=\left(\frac{1}{D a}-R v h_{2}^{2}\right) \cosh \left(h_{4}\right),
$$$$
r_{30}=\left(\frac{1}{D a}-R v h_{2}^{2}\right) \sinh \left(h_{4}\right), \quad r_{31}=h_{7} e^{h_{6}} \cos \left(h_{6}\right),
$$$$
r_{32}=h_{7} e^{h_{6}} \sin \left(h_{6}\right) \text {, }
$$$$
r_{33}=h_{7} e^{-h_{6}} \sin \left(h_{6}\right) \text {, }
$$$$
r_{35}=N h_{8} e^{h_{6}}\left(\cos \left(h_{6}\right)-\sin \left(h_{6}\right)\right) \text {, }
$$$$
r_{36}=N h_{8} e^{h_{6}}\left\{\cos \left(h_{6}\right)+\sin \left(h_{6}\right)\right\} \text {, }
$$$$
r_{37}=N h_{8} e^{-h_{6}}\left\{\cos \left(h_{6}\right)-\sin \left(h_{6}\right)\right\},
$$$$
r_{38}=N h_{8} e^{-h_{6}}\left(\cos \left(h_{6}\right)+\sin \left(h_{6}\right)\right) \text {, }
$$$$
r_{39}=\left(-R v h_{1}^{3}+\frac{h_{1}}{D a}\right) R c \sinh \left(h_{3}\right) \text {, }
$$$$
r_{40}=\left(-R v h_{1}^{3}+\frac{h_{1}}{D a}\right) R c \cosh \left(h_{3}\right) \text {, }
$$$$
r_{41}=\left(-R v h_{2}^{3}+\frac{h_{2}}{D a}\right) R c \sinh \left(h_{4}\right),
$$$$
r_{42}=\left(-R v h_{2}^{3}+\frac{h_{2}}{D a}\right) R c \cosh \left(h_{4}\right) \text {, }
$$$$
r_{43}=-\frac{r_{2}}{r_{1}},
$$$$
r_{44}=-\frac{r_{3}}{r_{1}}, \quad r_{45}=-\frac{r_{4}}{r_{1}},
$$$$
r_{46}=r_{7}-r_{5}
$$$$
r_{47}=r_{8}+r_{6},
$$$$
r_{48}=r_{10}+r_{43} r_{9} \text {, }
$$$$
r_{49}=r_{11}+r_{44} r_{9} \text {, }
$$$$
r_{50}=r_{12}+r_{45} r_{9} \text {, }
$$$$
r_{51}=r_{21} r_{6} \text {, }
$$

$$
\begin{aligned}
& r_{52}=r_{15}-r_{13}, \quad r_{53}=r_{14}+r_{16}, \\
& r_{54}=r_{43} r_{17}+r_{18}, \quad r_{55}=r_{44} r_{17}+r_{19} \text {, } \\
& r_{56}=r_{45} r_{17}+r_{20}, \quad r_{57}=r_{21} r_{14} \text {, } \\
& r_{58}=r_{43} r_{22}+r_{23}, \quad r_{59}=r_{44} r_{22}+r_{24} \text {, } \\
& r_{60}=r_{45} r_{22}+r_{25}, \quad r_{61}=r_{43} r_{27}+r_{28}, \\
& r_{62}=r_{44} r_{27}+r_{29}, \quad r_{63}=r_{45} r_{27}+r_{30} \text {, } \\
& r_{64}=r_{32}+r_{33}, \quad r_{65}=r_{31}-r_{34} \text {, } \\
& r_{66}=r_{21} r_{31}, \quad r_{67}=r_{35}+r_{38}, \\
& r_{68}=r_{36}+r_{37} \\
& r_{69}=r_{43} r_{39}+r_{40}, \quad r_{70}=r_{44} r_{39}+r_{41} \text {, } \\
& r_{71}=r_{45} r_{39}+r_{42}, \quad A r_{1}=r_{21} r_{35} \text {, } \\
& r_{72}=\frac{r_{26}}{r_{58}}, \quad r_{73}=\frac{r_{59}}{r_{58}}, \\
& r_{74}=\frac{r_{60}}{r_{58}}, \\
& r_{75}=r_{73} r_{48}-r_{49}, \\
& r_{76}=r_{74} r_{48}-r_{50}, \\
& r_{77}=r_{51}-r_{72} r_{48} \\
& r_{79}=r_{74} r_{54}-r_{56} \text {, } \\
& r_{81}=r_{62}-r_{73} r_{61} \text {, } \\
& r_{83}=r_{66}+r_{72} r_{61} \text {, } \\
& r_{87}=\frac{r_{47}}{r_{46}} \text {, } \\
& r_{89}=\frac{r_{76}}{r_{46}}, \\
& r_{91}=r_{53}-r_{87} r_{52} \text {, } \\
& r_{93}=r_{79}-r_{89} r_{52} \text {, } \\
& r_{95}=r_{81}-r_{88} r_{64} \text {, } \\
& r_{97}=r_{65}-r_{87} r_{64} \text {, } \\
& r_{99}=r_{67}-r_{87} r_{68} \text {, } \\
& r_{101}=r_{85}-r_{89} r_{68} \text {, } \\
& r_{103}=\frac{r_{92}}{r_{91}} \text {, } \\
& r_{105}=\frac{r_{94}}{r_{91}}, \\
& r_{107}=r_{96}-r_{104} r_{97} \text {, } \\
& r_{109}=r_{100}-r_{103} r_{99} \text {, } \\
& r_{111}=r_{102}-r_{105} r_{99} \text {, } \\
& r_{113}=\frac{r_{108}}{r_{106}}, \\
& r_{114}=r_{110}-r_{112} r_{109}, \\
& r_{115}=r_{111}-r_{113} r_{109} \text {, } \\
& r_{116}=-\frac{r_{115}}{r_{114}}, \quad C_{8}=r_{116}, \\
& C_{7}=-\left(r_{112} C_{8}+r_{113}\right), \quad C_{6}=r_{72}-r_{73} C_{7}-r_{74} C_{8}, \\
& C_{5}=r_{43} C_{6}+r_{44} C_{7}+r_{45} C_{8} \text {, } \\
& C_{4}=-\left(r_{105}+r_{103} C_{7}+r_{104} C_{8}\right) \text {, } \\
& C_{3}=-\left(r_{90}+r_{87} C_{4}+r_{88} C_{7}+r_{89} C_{8}\right) \text {, } \\
& C_{2}=r_{21}+C_{4}, \quad C_{1}=-C_{3} \text {. }
\end{aligned}
$$

\section{Case 2:}

$$
\begin{array}{lll}
N=\sqrt{\frac{\sqrt{R a}}{2}} & S=\sqrt{1-\frac{4 R a R v D a^{2}}{R c}}, & m=\frac{1}{2 D a R v}, \\
r_{1}=1 / 2 N^{2}, & r_{2}=\cosh m, & r_{3}=\sinh m, \\
r_{4}=m^{2} \cosh m, & r_{5}=m^{2} \cosh m+2 m \sinh m,
\end{array}
$$


N. Srivastava and A. K. Singh / JAFM, Vol. 3, No. 2, pp. 65-75, 2010.

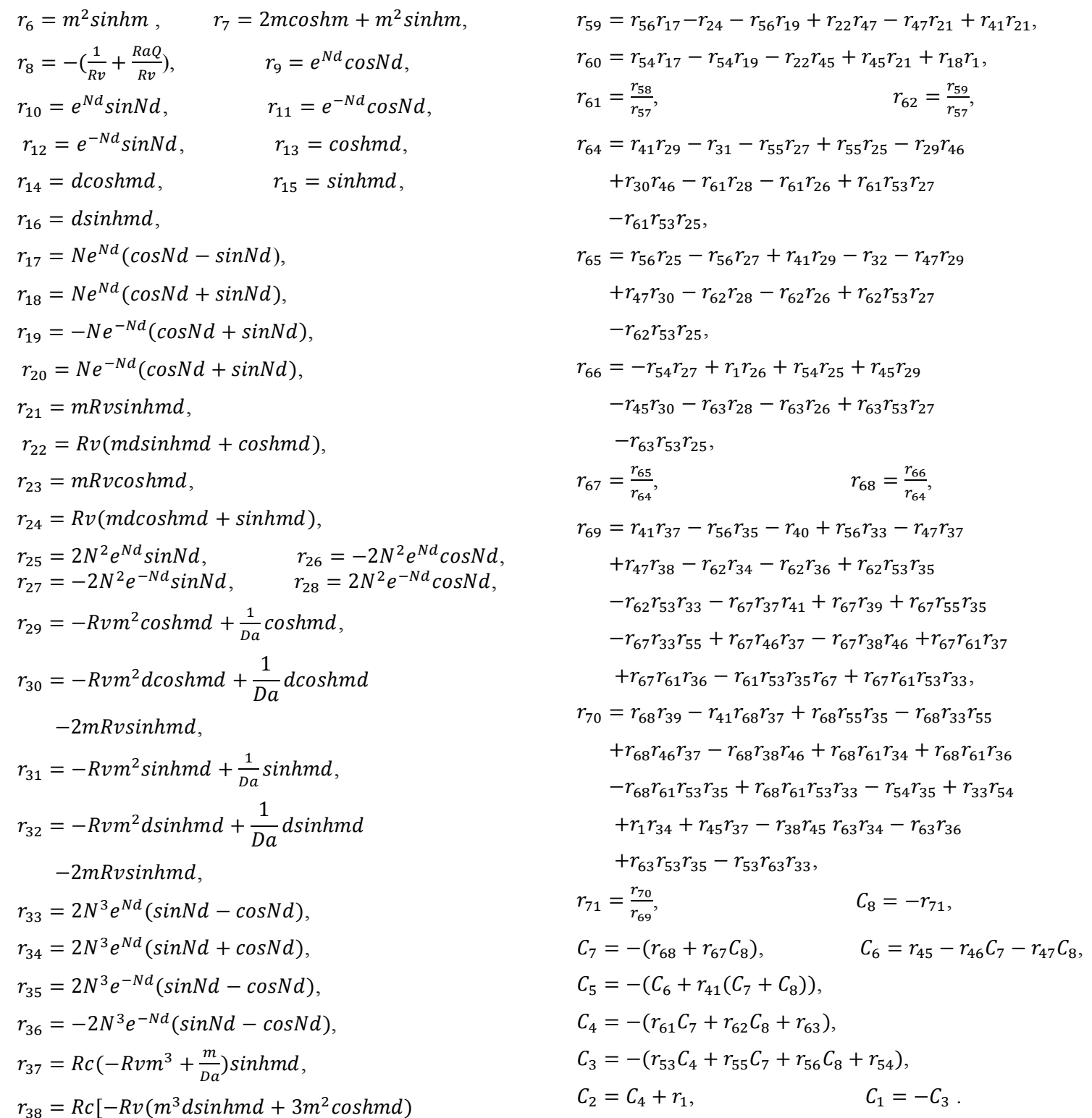

$\left.+\frac{m}{D a}(m d \sinh m d+\cosh m d)\right]$,

$r_{39}=R c\left(-R v m^{3}+\frac{m}{D a}\right) \operatorname{coshmd}$,

$r_{40}=R c\left[-R v\left(m^{3} d \operatorname{coshmd}+3 m^{2} \sinh m d\right)\right.$

$\left.+\frac{m}{D a}(\sinh m d+m d \cosh m d)\right]$,

$r_{41}=\frac{r_{3}}{r_{2}}, \quad r_{42}=r_{5}-r_{4}, \quad r_{43}=r_{6}-r_{4} r_{41}$,

$r_{44}=r_{7}-r_{4} r_{41}, \quad r_{45}=\frac{r_{8}}{r_{42}}, \quad r_{46}=\frac{r_{43}}{r_{42}}$,

$r_{47}=\frac{r_{44}}{r_{42}}, \quad r_{48}=r_{11}-r_{9}, \quad r_{49}=r_{12}-r_{10}$,

$r_{50}=r_{1} r_{10}+r_{45} r_{13}-r_{45} r_{14}$,

$r_{51}=r_{41} r_{13}-r_{46} r_{13}+r_{46} r_{14}-r_{15}$,

$r_{52}=r_{41} r_{13}-r_{47} r_{13}+r_{47} r_{14}-r_{16}$,

$r_{53}=\frac{r_{49}}{r_{48}}, \quad r_{54}=\frac{r_{50}}{r_{48}}, \quad r_{55}=\frac{r_{51}}{r_{48}}$,

$r_{56}=\frac{r_{52}}{r_{48}}, \quad r_{57}=r_{20}+r_{18}-r_{53} r_{19}+r_{53} r_{17}$,

$r_{58}=r_{55} r_{17}-r_{23}-r_{55} r_{19}+r_{46} r_{22}-r_{46} r_{21}+r_{41} r_{21}$,

Case 3:

$R=\sqrt{\frac{4 R a R v D a^{2}}{R c}-1}, \quad d_{1}=\sqrt{\frac{\sqrt{1+R^{2}}+1}{2}}$,

$d_{2}=\sqrt{\frac{\sqrt{1+R^{2}}-1}{2},} \quad \alpha=\frac{d_{1}}{\sqrt{2 D a R v}}$,

$\beta=\frac{d_{2}}{\sqrt{2 \text { DaRv }}}, \quad t=\sqrt{\frac{\sqrt{R a}}{2}}$,

$r_{1}=\frac{1}{2 N^{2}}, \quad r_{2}=e^{\alpha} \cos \beta$,

$r_{3}=e^{\alpha} \sin \beta, \quad r_{4}=e^{-\alpha} \cos \beta$,

$r_{5}=e^{-\alpha} \sin \beta$,

$r_{6}=\frac{e^{\alpha}}{D a R a} \cos \beta-\frac{R v}{R a} e^{\alpha}\left[\left(\alpha^{2}-\beta^{2}\right) \cos \beta-2 \alpha \beta \sin \beta\right]$,

$r_{7}=\frac{e^{\alpha}}{D a R a} \sin \beta-\frac{R v}{R a} e^{\alpha}\left[\left(\alpha^{2}-\beta^{2}\right) \sin \beta+2 \alpha \beta \cos \beta\right]$,

$r_{8}=\frac{e^{-\alpha}}{D a R a} \cos \beta-\frac{R v}{R a} e^{-\alpha}\left[\left(\alpha^{2}-\beta^{2} \cos \beta+2 \alpha \beta \sin \beta\right]\right.$, 
N. Srivastava and A. K. Singh / JAFM, Vol. 3, No. 2, pp. 65-75, 2010.

$$
\begin{array}{lrl}
r_{9}=\frac{e^{-\alpha}}{D a R a} \sin \beta-\frac{R v}{R a} e^{-\alpha}\left[\left(\alpha^{2}-\beta^{2}\right)\right. & \sin \beta-2 \alpha \beta \cos \beta], \\
r_{10}=\frac{T_{1}-T_{0}}{A H Q}-\frac{1}{R a}, & r_{11}=e^{N d} \cos N d, \\
r_{12}=e^{N d} \sin N d, & r_{13}=e^{-N d} \cos N d, \\
r_{14}=e^{-N d} \sin N d, & r_{15}=e^{\alpha d} \cos \beta d, \\
r_{16}=e^{\alpha d} \sin \beta d, & r_{17}=e^{-\alpha d} \cos \beta d, \\
r_{18}=e^{-\alpha d} \sin \beta d, & r_{19}=\frac{2 N^{2}}{R a} e^{N d} \sin N d, \\
r_{20}=-\frac{2 N^{2}}{R a} e^{N d} \cos N d, & \\
r_{21}=-\frac{2 N^{2}}{R a} e^{-N d} \sin N d, & \\
r_{22}=\frac{2 N^{2}}{R a} e^{-N d} \cos N d, & \\
r_{23}=\frac{e^{\alpha d}}{D a R a} \cos \beta-\frac{R v}{R a} e^{\alpha d}\left[\left(\alpha^{2}-\beta^{2}\right)\right. & \\
& \cos \beta d-2 \alpha \beta \sin \beta d], &
\end{array}
$$$$
r_{24}=\frac{e^{\alpha}}{D a R a} \sin \beta d-\frac{R v}{R a} e^{\alpha d}\left[\left(\alpha^{2}-\beta^{2}\right)\right.
$$$$
\sin \beta d+2 \alpha \beta \cos \beta d],
$$$$
r_{25}=\frac{e^{-\alpha d}}{D a R a} \cos \beta \mathrm{d}-\frac{R v}{R a} e^{-\alpha d}\left[\left(\alpha^{2}-\beta^{2}\right)\right.
$$$$
\cos \beta d+2 \alpha \beta \sin \beta d],
$$$$
r_{26}=\frac{e^{-\alpha d}}{D a R a} \sin \beta d-\frac{R v}{R a} e^{-\alpha d}\left[\left(\alpha^{2}-\beta^{2}\right)\right.
$$$$
\sin \beta d-2 \alpha \beta \cos \beta d] \text {, }
$$$$
r_{27}=N e^{N d}(\cos N d-\sin N d),
$$$$
r_{28}=N e^{N d}(\cos N d+\sin N d) \text {, }
$$$$
r_{29}=-N e^{-N d}(\cos N d+\sin N d) \text {, }
$$$$
r_{30}=N e^{-N d}(\cos N d-\sin N d) \text {, }
$$$$
r_{31}=R v e^{\alpha d}(\alpha \cos \beta d-\beta \sin \beta d),
$$$$
r_{32}=R v e^{\alpha d}(\beta \cos \beta d+\alpha \sin \beta d),
$$$$
r_{33}=-R v e^{-\alpha d}(\alpha \cos \beta d+\beta \sin \beta d) \text {, }
$$$$
r_{34}=-R v e^{-\alpha d}(\beta \cos \beta d-\alpha \sin \beta d) \text {, }
$$$$
r_{35}=\frac{2 N^{3}}{R a} e^{N d}(\cos N d+\sin N d) \text {, }
$$$$
r_{36}=\frac{2 N^{3}}{R a} e^{N d}(\sin N d-\cos N d),
$$$$
r_{37}=\frac{2 N^{3}}{R a} e^{-N d}(\sin N d-\cos N d),
$$$$
r_{38}=-\frac{2 N^{3}}{R a} e^{N d}(\cos N d+\sin N d),
$$$$
r_{39}=R c e^{\alpha d}\left\{\frac{1}{D a R a}(\alpha \cos \beta d-\beta \sin \beta d)-\frac{R v}{R a}\right.
$$$$
\left.\left[\left(\beta^{3}-3 \alpha^{2} \beta\right) \sin \beta d+\left(\alpha^{3}-3 \alpha \beta^{2}\right) \cos \beta d\right]\right\},
$$$$
r_{40}=R c e^{\alpha d}\left\{\frac{1}{D a R a}(\beta \cos \beta d+\alpha \sin \beta d)-\frac{R v}{R a}\right.
$$$$
\left.\left[\left(3 \alpha^{2} \beta-\beta^{3}\right) \cos \beta d+\left(\alpha^{3}-3 \alpha \beta^{2}\right) \sin \beta d\right]\right\},
$$$$
r_{41}=\left\{-R c e^{-\alpha d}\left\{\frac{1}{D a R a}(\alpha \cos \beta d+\beta \sin \beta d)\right)-\frac{R v}{R a}\right.
$$$$
\left.\left[\left(\beta^{3}-3 \alpha^{2} \beta\right) \sin \beta d+\left(3 \alpha \beta^{2}-\alpha^{3}\right)+\cos \beta d\right]\right\},
$$$$
r_{42}=R c e^{-\alpha d}\left[-\frac{1}{D a R a}(\beta \cos \beta d-\alpha \sin \beta d)-\frac{R v}{R a}\right.
$$$$
\left.\left\{\left(3 \alpha^{2} \beta-\beta^{3}\right) \cos \beta d+\left(3 \alpha \beta^{2}-\alpha^{3}\right)+\sin \beta d\right\}\right],
$$$$
r_{43}=\frac{r_{3}}{r_{2}}, \quad r_{44}=\frac{r_{4}}{r_{2}}, \quad r_{45}=\frac{r_{5}}{r_{2}},
$$

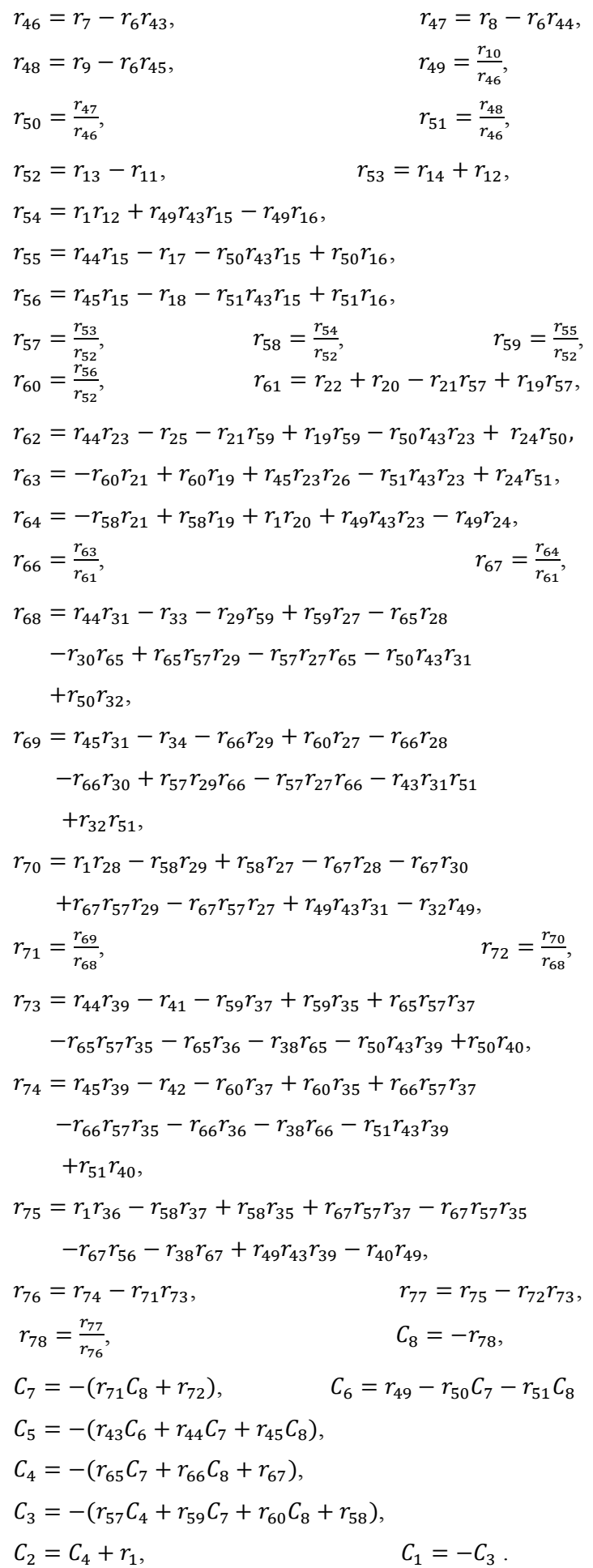

